\title{
Symptomatic Intracranial Hemorrhage following Intravenous Thrombolysis for Acute Ischemic Stroke: A Critical Review of Case Definitions
}

\author{
Raymond C.S. Seet ${ }^{a, b}$ Alejandro A. Rabinstein ${ }^{a}$ \\ a Department of Neurology, Mayo Clinic, Rochester, Minn., USA; b Department of Medicine, Yong Loo Lin School of \\ Medicine, National University of Singapore, Singapore
}

\section{Key Words}

Recombinant tissue plasminogen activator $\cdot$ Ischemic stroke $\cdot$ Symptomatic intracranial hemorrhage

\begin{abstract}
Background: Symptomatic intracranial hemorrhage ( $\mathrm{SICH})$ is a devastating complication of intravenous thrombolysis treatment that is associated with high mortality. Clinical trials, stroke registries and cohort studies employ different case definitions to identify stroke patients with SICH following intravenous thrombolysis. We systematically reviewed the reported rates of $\mathrm{SICH}$ following intravenous thrombolysis and compared their consistency with mortality outcomes. Methods: Studies were identified from the PubMed and Embase databases from January 1994 to July 2011 by cross-referencing the following MeSH terms: 'thrombolysis', 'recombinant tissue plasminogen activator', 'rtPA', 'hemorrhagic stroke', 'cerebral hemorrhage', 'hematoma' and 'ischemic stroke'. Demographic information, baseline National Institute of Health Stroke Scale (NIHSS) scores, time from stroke onset to intravenous thrombolysis, $\mathrm{SICH}$ and mortality rates were derived from published data in 7 randomized controlled trials, 7 stroke registries and 10 cohort studies ( 4 multicenter and 6 single center) with more than 200 consecutively recruited patients. Mortality rates were considered as the percentage of patients treated with intravenous thrombolysis who died within 90 days after stroke. Results: The mean age of patients included in this analysis was 68.8 years
\end{abstract}

(standard deviation, SD 2.9, range 63-75), of whom $56.3 \%$ (SD 4.5, range 45-63) were men. They presented with a mean baseline NIHSS of 12.5 (SD 1.4, range 9-15) and received intravenous thrombolysis $175 \mathrm{~min}$ (SD 62, range 120-328) from stroke onset. The overall mean $\mathrm{SICH}$ and mortality rates of patients treated with intravenous thrombolysis were 5.6\% (SD 2.3) and 14.7\% (SD 4.8), respectively. A moderate correlation was observed between the incidence of $\mathrm{SICH}$ and mortality in patients treated with intravenous thrombolysis $(r=$ $0.401, p=0.050$ ). The variation in $\mathrm{SICH}$ rates was highest across studies that reported $\mathrm{SICH}$ rates using the Safe Implementation of Thrombolysis in Stroke-Monitoring Study (SITS-MOST) criteria compared with the European Cooperative Acute Stroke Study and National Institute of Neurological Disorders and Stroke (NINDS) criteria. Studies that defined $\mathrm{SICH}$ as parenchymal hemorrhage with a neurological decline NIHSS $\geq 4$ occurring within $36 \mathrm{~h}$ of intravenous thrombolysis reported a higher consistency between $\mathrm{SICH}$ and mortality rates (correlation coefficient 0.631 ). Conclusions: $\mathrm{SICH}$ rates vary considerably between studies and these differences may relate to the differences in the criteria used to define $\mathrm{SICH}$. Until a case definition with high interrater agreement and good correlation with stroke outcomes becomes available, detailed information on the type of bleeding, the extent of NIHSS deterioration, neuroimaging features and the time from thrombolysis to diagnosis of hemorrhage should be reported to permit a correct interpretation of $\mathrm{SICH}$ rates.

Copyright $\odot 2012$ S. Karger AG, Basel

\section{KARGER}

Fax +4161306 1234

E-Mail karger@karger.ch

www.karger.com
(C) 2012 S. Karger AG, Basel

$1015-9770 / 12 / 0342-0106 \$ 38.00 / 0$

Accessible online at:

www.karger.com/ced
Dr. Raymond Chee Seong Seet

Department of Medicine, National University Health System

$1 \mathrm{E}$, NUHS Tower Block, Kent Ridge Road

Singapore 119228 (Singapore)

Tel. +65 6779 5555, E-Mail raymond_seet@ nus.edu.sg 


\section{Introduction}

Differences exist in the criteria used to define the neurological decline, cerebral hemorrhage and interval between intravenous thrombolysis and the onset of cerebral hemorrhage (online suppl. table 1 ; for all online suppl. material, see www.karger.com/doi/10.1159/000339675). The initial investigators of the National Institute of Neurological Disorders and Stroke (NINDS) studies considered symptomatic hemorrhage in patients as 'contemporaneous neurological worsening' [1], while subsequent NINDS studies defined symptomatic intracranial hemorrhage $(\mathrm{SICH})$ as 'any CT-documented hemorrhage that was temporally related to deterioration in the patient's clinical condition in the judgment of the clinical investigator' [2].

Due to the high frequency of clinically irrelevant cerebral hemorrhage after intravenous thrombolysis, an additional criterion to quantify the extent of clinical deterioration was adopted by investigators of the Prolyse for Acute Cerebral Thromboembolism (PROACT) II study [3], the European Cooperative Acute Stroke Study (ECASS) [4-6] and the Safe Implementation of Thrombolysis in Stroke-Monitoring Study (SITS-MOST) [7] studies; a 4-point change in the National Institute of Health stroke scale (NIHSS) was thought unlikely to be explained by random fluctuation in scoring between investigators. By comparison, investigators of Diffusion and Perfusion Imaging Evaluation for Understanding Stroke Evolution (DEFUSE) distinguished between 'minor' symptomatic hemorrhage (patients with a 2- to 3point increment in the NIHSS) and 'major' hemorrhage (patients with $\geq 4$-point increment) [8].

Although both the NINDS and the ECASS studies distinguished cerebral hemorrhage according to petechial (isolated or confluent) and hematoma patterns, these neuroimaging criteria differed by the inclusion of mass effect in the ECASS but not in the NINDS studies $[2,6]$. The NINDS, SITS-MOST and DEFUSE definitions considered hemorrhages attributable to thrombolysis when they occurred within $36 \mathrm{~h}[2,7,9,10]$, while ECASS II considered hemorrhages to be of clinical importance when they occurred within 7 days of intravenous thrombolysis [5]. There is an increasing trend for trials and studies to report multiple SICH rates using different definitions (tables 1-3).

We performed a systematic review to compare the incidence of SICH following intravenous thrombolysis in selected randomized clinical trials, stroke registries and cohort studies, and to appraise the criteria used to define $\mathrm{SICH}$ in relation to the mortality rates reported in the literature.

\section{Methods}

Search Strategy and Selection Criteria

Studies were identified from the PubMed and Embase databases from January 1994 to July 2011 by cross-referencing the following MeSH terms: 'thrombolysis', 'recombinant tissue plasminogen activator', 'rtPA', 'hemorrhagic stroke', 'cerebral hemorrhage', 'hematoma' and 'ischemic stroke'. Further studies were identified from the reference lists, related articles and citation lists of each of the papers identified in the initial searches. We included major randomized controlled trials, stroke registries and cohort studies with designs that allowed a calculation of age, baseline NIHSS scores, interval between stroke onset and intravenous thrombolysis, SICH and mortality rates. Our selection was restricted to stroke registries and larger cohort studies that comprised consecutively recruited stroke patients with an operational cutoff of $>200$ patients. Studies that included patients treated endovascularly and that applied preselection criteria (e.g. the presence of demonstrable arterial occlusion and availability of pretreatment MRI), and which did not report both SICH and mortality rates, were excluded. Only papers published in English were included.

\section{Data Extraction}

Two authors (R.C.S.S. and A.A.R.) performed the data search and quality assessment independently and completed a data extraction form. The case definitions used to define SICH, in terms of neurological decline, neuroimaging criteria and interval between intravenous thrombolysis and development of SICH, were analyzed.

\section{Statistical Analysis}

The mean age, baseline NIHSS scores and time from stroke onset to intravenous thrombolysis were computed for each study. The SICH and mortality rates for these studies were pooled by the use of a random-effects binomial meta-analysis; random-effects models were used because of heterogeneity in the incidence between studies. To study sources of heterogeneity of incidence, subgroup analyses and binomial metaregression were performed. Mortality rates were considered as the percentage of patients treated with intravenous thrombolysis who died within 90 days after stroke. The proportion of patients with poor functional recovery could not be computed due to the lack of standardized cutoffs used to define good and poor stroke outcomes. We compared the correlation coefficients between SICH and mortality rates to assess the consistency of SICH across these studies. Summary data from studies that defined SICH using either the SITSMOST, ECASS or NINDS criteria were pooled to separately compare the mean, standard deviation (SD) and variance of SICH rates according to these criteria. Using mean values of these pooled data, a conversion factor of SICH rates was calculated by considering the ratios of the 'target' and 'original' case definitions of SICH. SPSS 17.0 software (SPSS Inc., Chicago, Ill., USA) was used for all statistical analyses, except for the random-effects binomial regression, for which SAS 9.1 (SAS Institute Inc., Cary, N.C., USA) was used. 
Table 1. Randomized controlled trial data of stroke patients treated with intravenous recombinant tissue plasminogen activator

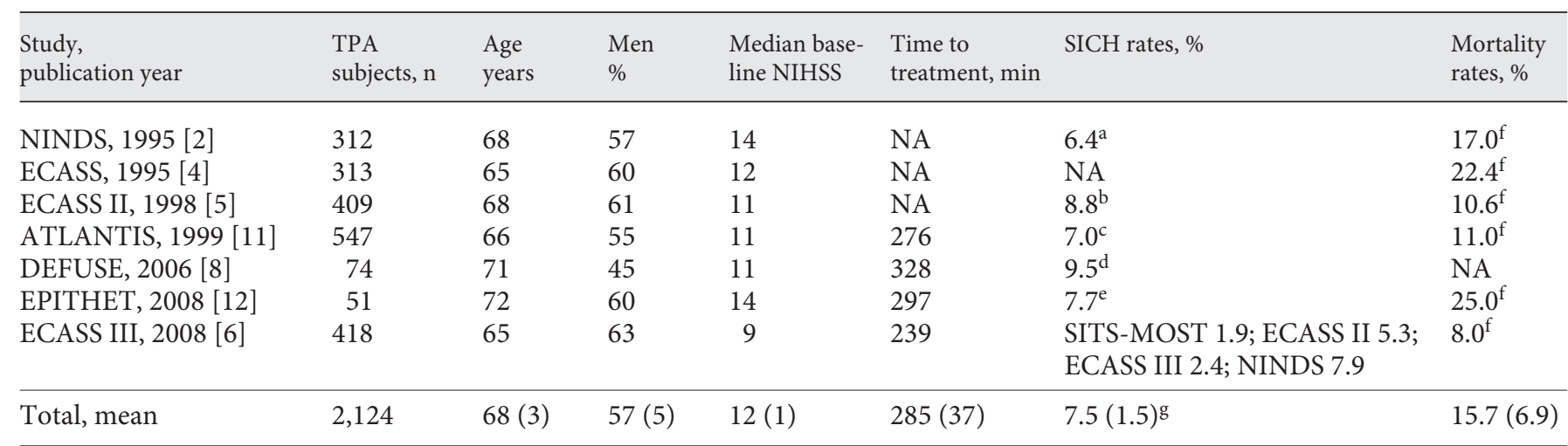

Data are summarized as mean or median and values in parentheses indicate $\mathrm{SD}$. TPA = Tissue plasminogen activator; ATLANTIS = Alteplase Thrombolysis for Acute Noninterventional Therapy in Ischemic Stroke; EPITHET = Echoplanar Imaging Thrombolysis Evaluation Trial; NA = not available.

${ }^{a}$ NINDS criteria (any neurologic decline, any cerebral hemorrhage and $<36 \mathrm{~h}$ following intravenous thrombolysis). ${ }^{\mathrm{b}}$ ECASS II criteria (any neurologic decline, clinical worsening or NIHSS de- cline $\geq 4$, any cerebral hemorrhage and $<7$ days following intravenous thrombolysis). ${ }^{\mathrm{c}}$ Not available. ${ }^{\mathrm{d}}$ DEFUSE (NIHSS decline $\geq 2$, any cerebral hemorrhage and $<36 \mathrm{~h}$ following intravenous

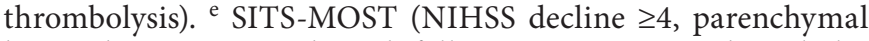
hemorrhage type 2 and $<36 \mathrm{~h}$ following intravenous thrombolysis). ${ }^{\mathrm{f}} 90$ days after stroke. ${ }^{\mathrm{g}}$ ECASS II data were used in studies that reported $>1$ SICH rates.
Fig. 1. Literature search and selection of studies.

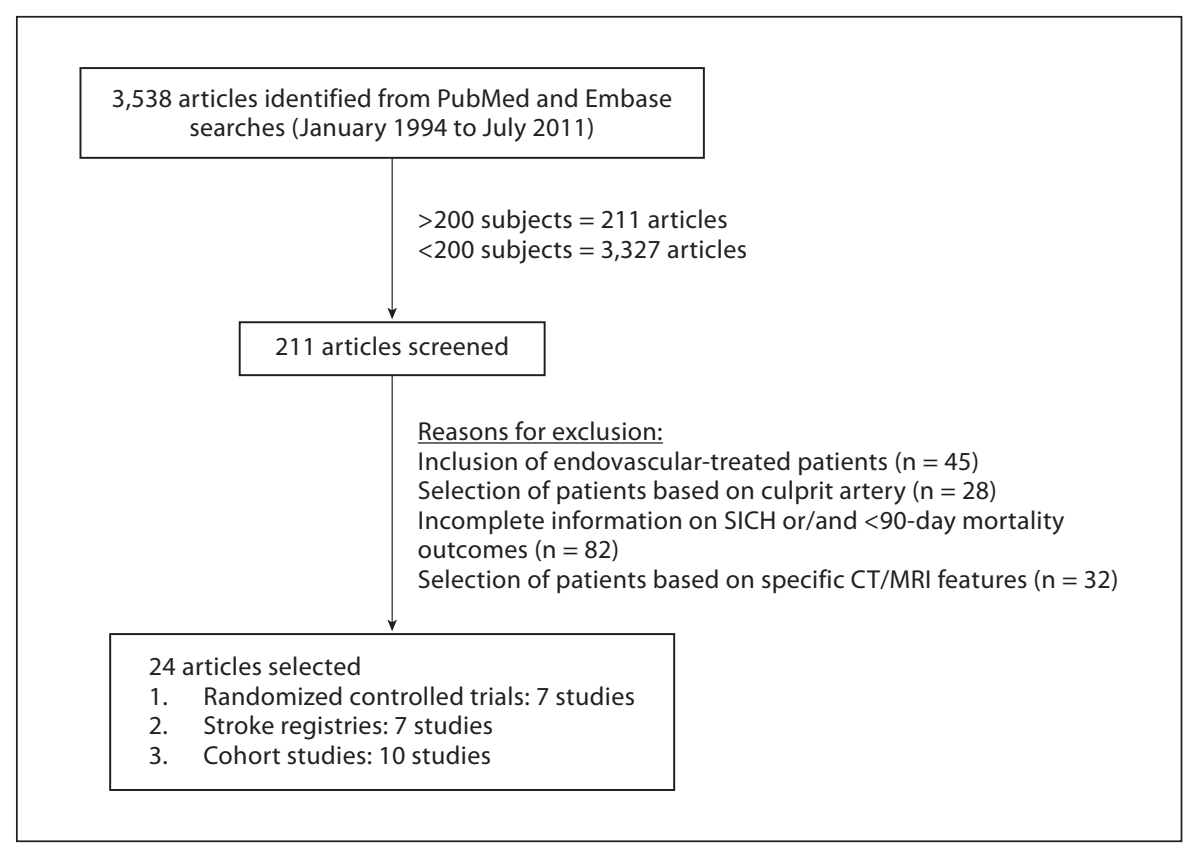

\section{Results}

\section{Study Identification and Selection}

The initial search identified 3,538 nonduplicate studies which were reduced to 211 potentially eligible studies that were relevant to our investigation (fig. 1). After the application of the exclusion criteria, 7 clinical trials $[2$, $4-6,8,11,12], 7$ stroke registries $[7,9,10,13-16]$ and 10 cohort studies (4 multicenter [17-20] and 6 single-center [21-25]) were included in this analysis. Tables 1-3 describe the study population, baseline stroke severity, stroke onset to treatment time, $\mathrm{SICH}$ and mortality rates 
Table 2. Registry data of stroke patients treated with intravenous recombinant tissue plasminogen activator

\begin{tabular}{|c|c|c|c|c|c|c|c|}
\hline STARS, $2000[13]$ & 389 & 69 & 55 & 13 & 164 & $3.3^{\mathrm{a}}$ & $13.0^{\mathrm{d}}$ \\
\hline SITS-MOST, 2007 [7] & 6,483 & 68 & 60 & 12 & 136 & $\begin{array}{l}\text { SITS-MOST 1.7; ECASS } 4.6 \\
\text { ECASS II } 8.8 ; \text { NINDS } 7.3\end{array}$ & $11.2^{\mathrm{e}}$ \\
\hline SITS-ITSR, 2008 [9] & 12,529 & 68 & 61 & 12 & 143 & $\begin{array}{l}\text { SITS-MOST 1.6; ECASS } 4.8 \\
\text { NINDS } 7.3\end{array}$ & $12.1^{\mathrm{e}}$ \\
\hline SITS-ITSR, 2010 [10] & 23,942 & 68 & 60 & 12 & 146 & $\begin{array}{l}\text { SITS-MOST } 1.75 ; \\
\text { ECASS } 4.85 ; \text { NINDS } 7.13\end{array}$ & $12.3^{\mathrm{e}}$ \\
\hline GWTG, 2011 [15] & 25,504 & 70 & 51 & 12 & 129 & $5.4^{\mathrm{a}}$ & $9.9^{f}$ \\
\hline Canadian Stroke Network, 2011 [16] & 1,739 & 75 & 51 & 12 & 145 & $5.9^{\mathrm{c}}$ & $16.3^{\mathrm{f}}$ \\
\hline Total, mean & 15,054 & $70(3)$ & $56(4)$ & $12(1)$ & $145(12)$ & $3.5(1.8)^{\mathrm{g}}$ & $13.9(4.2)$ \\
\hline
\end{tabular}

following intravenous thrombolysis in these studies. As expected, stroke registries included the largest number of patients compared with randomized controlled trials and cohort studies. The mean age of patients was 68.8 years (SD 2.9, range 63-75), of whom 56.3\% (SD 4.5, range 4563) were men. These patients presented with baseline NIHSS of 12.5 (SD 1.4, range 9-15) and received intravenous thrombolysis $175 \mathrm{~min}$ (SD 62, range 120-328) from stroke onset (tables 1-3).

Although several randomized studies had a central adjudication process to assign SICH in patients with cerebral hemorrhage $[5,6,8]$, the decision to ascribe SICH was left to the discretion of individual investigators in the majority of the studies $[2,11,12]$. Among stroke registries, only one national registry, the Canadian Alteplase for Stroke Effectiveness Study (CASES), included central adjudication of SICH [14], and one cohort study had central adjudication of patients with suspected SICH [24]. Details of the change in the NIHSS before and after the onset of SICH were not provided in these studies. Four studies reported the mortality rates associated with SICH (mean $67.4 \%$, range $54-$ $79)[13,14,17,24]$. Depending on the criteria used to define $\mathrm{SICH}$, the mortality rate of patients with $\mathrm{SICH}$ ranged from 42 to $62 \%$; the mortality rate was higher when SICH was defined using the SITS-MOST criteria and lower when defined using the NINDS criteria [24].

\section{Case Definitions of Neurological Decline}

Each of the randomized clinical trials listed in table 1 employed unique case definitions of SICH $[2,4-6,8,11$, 12]. The ECASS II [5], ECASS III [6], DEFUSE [8] and EPITHET [12] trials specified the extent of neurological decline that needed to be present before a hemorrhage was considered to be 'symptomatic', while the NINDS trial did not provide such specification [2]. The methods of SICH ascertainment were not elaborated in the ECASS [4] and ATLANTIS [11] studies. On the other hand, the ECASS III trial reported the incidence of SICH using the different definitions adopted by the SITS-MOST, ECASS II, ECASS III and NINDS studies [6].

Similarly, stroke registries and cohort studies differed in their definitions of SICH. Both CASES [14] and the Canadian Stroke Network [16] did not specify the extent of neurological decline for a hemorrhage to be considered symptomatic, while the SITS-MOST [7] and SITS-ITSR studies $[9,10]$ required an NIHSS decline $\geq 4$ points to qualify as SICH. The methods of SICH determination were not explicit in the STARS [8] and GWTG [15] studies. In patients with cerebral hemorrhage, several cohort studies specified NIHSS decline $\geq 4$ points to indicate significant neurological deterioration $[19,21,25,26]$, while others considered any form of neurological decline to be significant $[22,23]$. To date, only the SITS-MOST [7] and SITS-ITSR $[9,10]$ registries and two cohort stud- 
Table 3. Cohort data of stroke patients treated with intravenous recombinant tissue plasminogen activator

\begin{tabular}{|c|c|c|c|c|c|c|c|}
\hline Study, publication year & $\begin{array}{l}\text { TPA } \\
\text { subjects, } \mathrm{n}\end{array}$ & $\begin{array}{l}\text { Age } \\
\text { years }\end{array}$ & $\begin{array}{l}\text { Men } \\
\%\end{array}$ & $\begin{array}{l}\text { Median base- } \\
\text { line NIHSS }\end{array}$ & $\begin{array}{l}\text { Time to treat- } \\
\text { ment, min }\end{array}$ & SICH rates, \% & $\begin{array}{l}\text { Mortality } \\
\text { rates, \% }\end{array}$ \\
\hline Tanne et al., 2002 [17] & 1,205 & 67 & 56 & NA & NA & $6.0^{\mathrm{a}}$ & $13.5^{\mathrm{f}}$ \\
\hline Berrouschot et al., 2005 [19] & 228 & 68 & 61 & 14 & NA & $2.6^{\mathrm{b}}$ & $7.9^{g}$ \\
\hline Chao et al., 2010 [20] & 241 & 66 & 60 & 15 & 139 & $\begin{array}{l}\text { SITS-MOST 3.7; ECASS 5.4; } \\
\text { NINDS } 7.9\end{array}$ & $10.0^{\mathrm{g}}$ \\
\hline Sobesky et al., 2007 [26] & 450 & 66 & 62 & 11 & 135 & $4^{\mathrm{d}}$ & $11^{\mathrm{g}}$ \\
\hline Uyttenboogaart et al., 2008 [23] & 252 & 68 & 54 & 12 & 174 & $5.2^{\mathrm{e}}$ & $17^{\mathrm{g}}$ \\
\hline Seet et al., 2011 [25] & 212 & 74 & 50 & 13 & 141 & $7.9^{\mathrm{b}}$ & $20^{\mathrm{h}}$ \\
\hline Strbian et al., 2011 [24] & 985 & 71 & 54 & 9 & 120 & $\begin{array}{l}\text { SITS-MOST 2.1; ECASS II } 7.0 \\
\text { NINDS } 9.4\end{array}$ & $10.2^{\mathrm{g}}$ \\
\hline
\end{tabular}

ies $[20,24]$ reported SICH rates using more than one case definition.

\section{Case Definitions of Cerebral Hemorrhage}

The NINDS, ECASS and DEFUSE studies considered any new cerebral hemorrhage to be significant in their definition of SICH $[2,4-6,8]$, while the SITS-MOST and SITS-ITSR studies considered only patients with parenchymal hemorrhage type $2[7,9,10]$ to be significant. Among the stroke registries, CASES considered only those with parenchymal hemorrhage [14] while the Canadian Stroke Network included patients with any cerebral hemorrhage following intravenous thrombolysis [16]. In several cohort studies, the presence of any hemorrhage after intravenous thrombolysis has been reported without accompanying details on neurological decline $[19,21-23,25,26]$. Although CT imaging was used to diagnose cerebral hemorrhage in a majority of these studies, both DEFUSE and EPITHET studies incorporated MR imaging in the evaluation of stroke patients with cerebral hemorrhage $[8,12]$. In the majority of the randomized controlled trials, neuroimaging assessors were blinded to the clinical outcomes $[2,5,6,8,12]$; this rigor, however, was adopted in only 1 stroke registry [14] and 2 cohort studies [24, 25].

\section{Interval between Intravenous Thrombolysis and Cerebral Hemorrhage}

Among randomized controlled trials, the ECASS II study reported any cerebral hemorrhage as that within 7 days of intravenous thrombolysis [2], while the DEFUSE and SITS-MOST studies reported hemorrhages as those diagnosed within the first $36 \mathrm{~h}$ after thrombolysis $[5,6$, $8,12]$. Although patients in the NINDS study were followed for up to 10 days, the interval used for its primary analysis was $36 \mathrm{~h}$ [2]. Stroke registries such as CASES, however, used a time interval of $24 \mathrm{~h}$ [14], while the Canadian Stroke Network and SITS-MOST and SITS-ITSR studies considered hemorrhages that occurred within 36 $\mathrm{h}$ to be associated with intravenous thrombolysis $[7,9,10$, 16]. Similar differences exist in the cohort studies, where hemorrhages occurring $<36 \mathrm{~h}[19,22,23]$ and $<48 \mathrm{~h}$ [23] following thrombolysis were considered to be causally linked to intravenous thrombolysis.

\section{Incidence of SICH and Mortality Rates}

The mean incidence of SICH following intravenous thrombolysis was $5.6 \%$ (SD 2.3) and the mean poststroke mortality rate was $14.7 \%$ (SD 4.8). Significant differences were observed in the incidence of SICH according to the study design; higher rates were reported in randomized 
Table 4. Comparison of mean and SD of symptomatic intracranial hemorrhage rates (defined according to the SITS-MOST, ECASS and NINDS criteria) among stroke patients following intravenous thrombolysis

\begin{tabular}{llll}
\hline & Mean SICH rates, $\%$ & SD, \% & Difference from mean SICH rates $(\%)$ \\
\hline $\begin{array}{l}\text { SITS-MOST criteria } \\
{[5-7,9,10,12,20,21,24]}\end{array}$ & 3.25 & 2.27 \\
ECASS criteria & 5.67 & 1.15 \\
{$[5-7,9,10,19,20,24,25]$} & 7.61 & 0.94 \\
NINDS criteria & & & -4 \\
{$[2,6,7,9,10,20,24]$} & &
\end{tabular}

Studies that reported SICH rates using either the SITS-MOST, ECASS or NINDS criteria were selected to calculate the mean values, SD and variance in SICH rates. Several studies [references 6, 7, 9, 10, 20, 24] reported SICH rates using all three criteria. Figures in the far right column illustrate the spread of SICH values from the calculated mean values.

controlled trials (mean 7.45\%) and lower rates were reported in stroke registries (mean 3.5\%). These differences, however, were not observed in the mortality rates of patients following intravenous thrombolysis across different study designs (online suppl. fig. 1A, B). In studies that reported SICH using different criteria $[6,7,9,10,20$, 24], there was a trend towards lower rates when SICH was defined using the SITS-MOST criteria compared with those studies that employed the NINDS criteria.

A lower incidence but a higher variability in $\mathrm{SICH}$ rates was observed across studies that employed the SITSMOST criteria whilst lower variability was found when SICH rates were reported using the NINDS criteria (table 4). Using mean values of SICH from studies that reported SICH rates according to divergent criteria, conversion factors between SICH rates across various case definitions were calculated and summarized in online supplementary table 2 . To assess the consistency of the different criteria used to define SICH, we compared the SICH and mortality rates across studies. Overall, a moderate correlation was observed between the incidence of SICH and mortality in patients treated with intravenous thrombolysis ( $\mathrm{r}=0.401, \mathrm{p}=0.050$; online suppl. fig. $1 \mathrm{C}$ ). The strengths of this relationship vary considerably across studies (online suppl. fig. 1D). Studies that incorporated the use of two or more of these criteria reported a higher consistency between SICH and mortality rates (table 5). Studies that defined SICH according to (1) parenchymal hemorrhage, (2) neurological decline NIHSS $\geq 4$ and (3) occurring within $36 \mathrm{~h}$ of intravenous thrombolysis had a greater consistency of association between SICH and mortality rates (table 5).

Intracranial Hemorrhage following Intravenous Thrombolysis
Table 5. Correlational analysis between components of SICH criteria and $<90$-day mortality rates after stroke

\begin{tabular}{|c|c|c|}
\hline & $\begin{array}{l}\text { Correlation } \\
\text { coefficient } r\end{array}$ & $\begin{array}{l}\mathrm{p} \\
\text { values }\end{array}$ \\
\hline \multicolumn{3}{|l|}{ Clinical criteria } \\
\hline Any ( $\mathrm{n}=9$ studies $)$ & -0.933 & 0.001 \\
\hline NIHSS decline $\geq 4$ ( $\mathrm{n}=20$ studies $)$ & 0.431 & 0.084 \\
\hline \multicolumn{3}{|l|}{ Neuroimaging criteria } \\
\hline Any ( $\mathrm{n}=20$ studies $)$ & 0.257 & 0.320 \\
\hline Parenchymal hemorrhage ( $\mathrm{n}=9$ studies) & 0.622 & 0.100 \\
\hline \multicolumn{3}{|l|}{ Time criteria } \\
\hline$<36 \mathrm{~h}(\mathrm{n}=21$ studies $)$ & 0.434 & 0.072 \\
\hline$<7$ days $(\mathrm{n}=4$ studies $)$ & -0.977 & 0.023 \\
\hline \multicolumn{3}{|l|}{ Number of criteria fulfilled $^{a}$} \\
\hline 1 criterion $(\mathrm{n}=4$ studies $)$ & -0.917 & 0.085 \\
\hline 2 criteria ( $\mathrm{n}=11$ studies $)$ & 0.467 & 0.205 \\
\hline 3 criteria $(\mathrm{n}=7$ studies $)$ & 0.631 & 0.128 \\
\hline
\end{tabular}

${ }^{\text {a }}$ Either (1) NIHSS decline $\geq 4$, (2) parenchymal hemorrhage or (3) time $<36 \mathrm{~h}$ following intravenous thrombolysis.

\section{Discussion}

$\mathrm{SICH}$ is a devastating complication of intravenous thrombolysis treatment that is associated with high mortality. Our study highlights two important observations: (1) the incidence of SICH following intravenous thrombolysis treatment varies considerably across stroke studies and according to the criteria used to define SICH, and (2) these differences resulted in marked inconsistencies between SICH and mortality rates. Data from this review suggest that the highest consistency was observed in the cohort studies and in the studies that defined SICH as 
parenchymal hemorrhage associated with NIHSS increments of $\geq 4$ points occurring within $36 \mathrm{~h}$ of intravenous thrombolysis. To our knowledge, this is the first systematic review that assesses the incidence of SICH among patients treated with intravenous thrombolysis. Previous narrative reviews [27-29] focused on data derived from randomized controlled trials and one meta-analysis included patients treated outside of the setting of clinical trials [30].

What is considered to be significant neurological decline differs across studies. The NINDS and ECASS II criteria considered any neurological decline to be significant, thus allowing for the inclusion of patients with mild and transient neurological worsening, which is fairly prevalent in the first couple of days after a stroke. Although the use of clinical severity scores to quantify the extent of neurological deterioration (e.g. NIHSS $\geq 4$ ) provides an objective assessment of hemorrhage severity, this method is limited by the 'ceiling effects' associated with these scores; patients with severe strokes and high baseline NIHSS scores may not exhibit a further increase in scores despite a significant hemorrhage. The determination of SICH using these quantitative criteria is demanding as it requires close (sometimes hourly) monitoring of NIHSS scores to identify patients at the onset of neurological decline, thus limiting its use to studies performed prospectively. Despite these technical considerations, the use of quantitative scoring to define neurological decline has been reported retrospectively in several studies [31, 32]. Furthermore, in patients with an insidious and progressive course of deterioration, such baseline levels may be difficult to establish. In ECASS II, when doubts arose as to whether edema or hemorrhage was the leading pathology, an association of the hemorrhage was assumed [5], whereas in ECASS III, adjudication of the cause of death or neurological decline (attributed to intracranial hemorrhage, other brain injury or neither of these causes) was performed by the chairs of the safety outcome adjudication committee and the steering committee [6].

Investigators of CASES have previously highlighted that not all sizable hemorrhages result in symptoms [33]. Massive bleedings into noneloquent areas of the brain may be asymptomatic or only slightly symptomatic (though these may potentially have an impact on the eventual chances of favorable functional recovery). These same bleedings (albeit small) may be symptomatic in strokes affecting a dominant cerebral hemisphere. Accumulating data indicate that the pattern and size of the hemorrhage, as well as associated features such as cere- bral edema, may be important in SICH determination [4-6]. The distinction of cerebral hemorrhages between hemorrhagic infarctions and parenchymal hemorrhages is pertinent because parenchymal hemorrhages that exceed $30 \%$ of the infarcted area with space-occupying effect are associated with increased risks of early neurological deterioration and higher 3-month mortality [34]. By contrast, although smaller hemorrhages do not generally have an impact on functional recovery, petechiae that are more confluent (termed 'HI2') are associated with worse stroke outcomes [34].

Several studies such as EPITHET and DEFUSE used $\mathrm{CT}$ and MRI scans interchangeably to report cerebral hemorrhage $[8,12]$. Despite data to suggest lower SICH and mortality rates in stroke patients selected for intravenous thrombolysis using MRI compared with CT modalities [35], the superiority of MR-based imaging has not been prospectively studied. It is not known whether the presence of leukoaraiosis and microbleeds detected on pretreatment MRI has any significance for SICH risks, and whether the more accurate characterization of hemorrhages afforded by sensitive imaging methods such as the gradient-recalled echo and the susceptibility-weighted imaging following intravenous thrombolysis have prognostic implications on hemorrhage risks and outcomes. Occasionally, retention of contrast may cause CT hyperdensity and changes on MRI such as $\mathrm{T}_{1}$-weighted hyperintensity, obscuring a clear distinction between cerebral hemorrhage and contrast staining following arterial recanalization.

The interval for SICH to be considered causally linked to intravenous thrombolysis varies across studies, ranging from $<24 \mathrm{~h}[14],<36 \mathrm{~h}[5-10,12,16,19,20,22,25]$, $<48 \mathrm{~h}[23]$ and $<7$ days $[2,6,7,9,10,20]$. Studies that report SICH according to the SITS-MOST criteria tended to report lower hemorrhagic rates compared to those that employed the ECASS criteria $[6,7,9,10,20,24]$. Despite manifesting initially as hemorrhagic infarctions, these hemorrhages may coalesce and transform into a parenchymal-type hemorrhage. An interval development of sepsis, cerebral edema and recurrent stroke may confound the assessment of neurological function associated with these hemorrhages. It is noteworthy that none of the studies included in this review reported the precise interval between intravenous thrombolysis and SICH development. This temporal information may be pertinent to determine causality, as cerebral hemorrhages that occur early are more likely to be attributed to intravenous thrombolysis compared to those that occur later. The variability in SICH rates (reported using different case 
definitions) may explain certain inconsistencies between $\mathrm{SICH}$ and mortality rates. Across the wide range of SICH definitions reported, the SICH rate explains only about one-sixth of the variation in mortality in recombinant tissue plasminogen activator-treated stroke patients. In ECASS III (the only randomized placebo-controlled study that reported SICH rates using different case definitions), fewer patients with cerebral hemorrhage in the placebo group were considered to have $\mathrm{SICH}$ when $\mathrm{SICH}$ was defined by ECASS III and SITS-MOST criteria (both $0.2 \%$ ) compared to ECASS II and NINDS criteria (2.2 and $3.5 \%$, respectively). These findings highlight inherent limitations of existing case definitions in describing thrombolysis-related hemorrhage.

Our study has several limitations. First, eligible articles were restricted to studies published in English and conference abstracts were not accessed. This may have resulted in the inadvertent exclusion of relevant studies in the non-English literature and unpublished data. Second, only cohort studies with $>200$ consecutive stroke patients were included and this inclusion criteria may have resulted in a selection of high-volume and experienced stroke centers. Third, to ensure uniformity in patient selection, only studies that included patients who received intravenous thrombolysis were chosen while those that reported patients undergoing endovascular treatment were excluded. Fourth, information included in this study was based on the published data; raw data were not available for analysis. It remains possible that these findings are in part due to a sampling error since small incidences are inevitably prone to greater variation.

This systematic study highlights several inconsistencies in the criteria used to define SICH. There is a pressing need for investigators to agree on the clinical, radiological and time interval criteria that should be used to define SICH and to standardize methods employed to report these outcomes. Until a case definition with a high interrater agreement rate and good correlation with clinical outcomes becomes available, information on the type of bleeding, the extent of NIHSS deterioration and the time interval between thrombolysis and bleeding should be reported to allow correct interpretation of SICH rates.

\section{Disclosure Statement}

Dr. Seet receives grants from the National Medical Research Council and National Research Foundation, Singapore.

Dr. Rabinstein has received research support from CardioNet Inc. and serves as safety monitor for a clinical trial funded by Boston Scientific.

The authors received no funding for this study.

\section{References}

1 Haley EC Jr, Levy DE, Brott TG, Sheppard GL, Wong MC, Kongable GL, et al: Urgent therapy for stroke. Part II. Pilot study of tissue plasminogen activator administered 91180 minutes from onset. Stroke 1992;23:641645.

- 2 The National Institute of Neurological Disorders and Stroke rt-PA Stroke Study Group: Tissue plasminogen activator for acute ischemic stroke. N Engl J Med 1995;333:15811587.

-3 Furlan A, Higashida R, Wechsler L, Gent M, Rowley H, Kase C, et al: Intra-arterial prourokinase for acute ischemic stroke. The PROACT-II study: a randomized controlled trial. Prolyse in acute cerebral thromboembolism. JAMA 1999;282:2003-2011.

-4 Hacke W, Kaste M, Fieschi C, Toni D, Lesaffre E, von Kummer R, et al: Intravenous thrombolysis with recombinant tissue plasminogen activator for acute hemispheric stroke. The European Cooperative Acute Stroke Study (ECASS). JAMA 1995;274: 1017-1025.
5 Hacke W, Kaste M, Fieschi C, von Kummer R, Davalos A, Meier D, et al: Randomised double-blind placebo-controlled trial of thrombolytic therapy with intravenous alteplase in acute ischaemic stroke (ECASSII). Second European-Australasian Acute Stroke Study investigators. Lancet 1998;352: 1245-1251.

-6 Hacke W, Kaste M, Bluhmki E, Brozman M, Davalos A, Guidetti D, et al: Thrombolysis with alteplase 3 to 4.5 hours after acute ischemic stroke. N Engl J Med 2008;359:13171329.

7 Wahlgren N, Ahmed N, Davalos A, Ford GA, Grond M, Hacke W, et al: Thrombolysis with alteplase for acute ischaemic stroke in the Safe Implementation of Thrombolysis in StrokeMonitoring Study (SITS-MOST): an observational study. Lancet 2007;369:275-282.

8 Albers GW, Thijs VN, Wechsler L, Kemp S, Schlaug G, Skalabrin E, et al: Magnetic resonance imaging profiles predict clinical response to early reperfusion: the Diffusion and Perfusion Imaging Evaluation for Understanding Stroke Evolution (DEFUSE) study. Ann Neurol 2006;60:508-517.
9 Wahlgren N, Ahmed N, Davalos A, Hacke W, Millan M, Muir K, et al: Thrombolysis with alteplase $3-4.5 \mathrm{~h}$ after acute ischaemic stroke (SITS-ISTR): an observational study. Lancet 2008;372:1303-1309.

10 Ahmed N, Wahlgren N, Grond M, Hennerici M, Lees KR, Mikulik R, et al: Implementation and outcome of thrombolysis with alteplase 3-4.5 $\mathrm{h}$ after an acute stroke: an updated analysis from SITS-ISTR. Lancet Neurol 2010;9:866-874.

-11 Clark WM, Wissman S, Albers GW, Jhamandas JH, Madden KP, Hamilton S: Recombinant tissue-type plasminogen activator (alteplase) for ischemic stroke 3 to 5 hours after symptom onset. The ATLANTIS study: a randomized controlled trial. Alteplase thrombolysis for acute noninterventional therapy in ischemic stroke. JAMA 1999;282: 2019-2026. 
12 Davis SM, Donnan GA, Parsons MW, LeviC, Butcher KS, Peeters A, et al: Effects of alteplase beyond $3 \mathrm{~h}$ after stroke in the Echoplanar Imaging Thrombolytic Evaluation Trial (EPITHET): a placebo-controlled randomised trial. Lancet Neurol 2008;7:299309.

13 Albers GW, Bates VE, Clark WM, Bell R, Verro P, Hamilton SA: Intravenous tissuetype plasminogen activator for treatment of acute stroke: the Standard Treatment with Alteplase to Reverse Stroke (STARS) study. JAMA 2000;283:1145-1150.

$\checkmark 14$ Hill MD, Buchan AM: Thrombolysis for acute ischemic stroke: results of the Canadian Alteplase for Stroke Effectiveness Study. CMAJ 2005;172:1307-1312.

-15 Fonarow GC, Smith EE, Saver JL, Reeves MJ, Bhatt DL, Grau-Sepulveda MV, et al: Timeliness of tissue-type plasminogen activator therapy in acute ischemic stroke: patient characteristics, hospital factors, and outcomes associated with door-to-needle times within 60 minutes. Circulation 2011;123: 750-758.

16 Vergouwen MD, Casaubon LK, Swartz RH, Fang J, Stamplecoski M, Kapral MK, Silver FL: Subtherapeutic warfarin is not associated with increased hemorrhage rates in ischemic strokes treated with tissue plasminogen activator. Stroke 2011;42:1041-1045.

17 Tanne D, Kasner SE, Demchuk AM, KorenMorag N, Hanson S, Grond M, et al: Markers of increased risk of intracerebral hemorrhage after intravenous recombinant tissue plasminogen activator therapy for acute ischemic stroke in clinical practice: the Multicenter rt-PA Stroke Survey. Circulation 2002;105:1679-1685.

18 Schenkel J, Weimar C, Knoll T, Haberl RL, Busse O, Hamann GF, et al: R1 - systemic thrombolysis in German stroke units - the experience from the German stroke data bank. J Neurol 2003;250:320-324.
19 Berrouschot J, Rother J, Glahn J, Kucinski T, Fiehler J, Thomalla G: Outcome and severe hemorrhagic complications of intravenous thrombolysis with tissue plasminogen activator in very old stroke patients. Stroke 2005; 36:2421-2425.

20 Chao AC, Hsu HY, Chung CP, Liu CH, Chen $\mathrm{CH}$, Teng MM, et al: Outcomes of thrombolytic therapy for acute ischemic stroke in Chinese patients: the Taiwan Thrombolytic Therapy for Acute Ischemic Stroke (TTTAIS) study. Stroke 2010;41:885-890.

21 Grotta JC, Burgin WS, El-Mitwalli A, Long M, Campbell M, Morgenstern LB, et al: Intravenous tissue-type plasminogen activator therapy for ischemic stroke: Houston experience 1996 to 2000. Arch Neurol 2001;58: 2009-2013.

-22 Ringleb PA, Schwark C, Kohrmann M, Kulkens S, Juttler E, Hacke W, et al: Thrombolytic therapy for acute ischaemic stroke in octogenarians: selection by magnetic resonance imaging improves safety but does not improve outcome. J Neurol Neurosurg Psychiatry 2007;78:690-693.

23 Uyttenboogaart M, Koch MW, Koopman K, Vroomen PC, Luijckx GJ, De Keyser J: Lipid profile, statin use, and outcome after intravenous thrombolysis for acute ischemic stroke. J Neurol 2008;255:875-880.

- 24 Strbian D, Sairanen T, Meretoja A, Pitkaniemi J, Putaala J, Salonen O, et al: Patient outcomes from symptomatic intracerebral hemorrhage after stroke thrombolysis. Neurology 2011;77:341-348.

25 Seet RC, Zhang Y, Moore SA, Wijdicks EF, Rabinstein AA: Subtherapeutic international normalized ratio in warfarin-treated patients increases the risk for symptomatic intracerebral hemorrhage after intravenous thrombolysis. Stroke 2011;42:2333-2335.

26 Sobesky J, Frackowiak M, Zaro-Weber O, Hahn M, Moller-Hartmann W, Rudolf J, et al: The Cologne stroke experience: safety and outcome in 450 patients treated with intravenous thrombolysis. Cerebrovasc Dis 2007;24:56-65.

-27 Derex L, Nighoghossian N: Intracerebral haemorrhage after thrombolysis for acute ischaemic stroke: an update. J Neurol Neurosurg Psychiatry 2008;79:1093-1099.
28 Thanvi BR, Treadwell S, Robinson T: Haemorrhagic transformation in acute ischaemic stroke following thrombolysis therapy: classification, pathogenesis and risk factors. Postgrad Med J 2008;84:361-367.

29 Trouillas P, von Kummer R: Classification and pathogenesis of cerebral hemorrhages after thrombolysis in ischemic stroke. Stroke 2006;37:556-561.

30 Graham GD: Tissue plasminogen activator for acute ischemic stroke in clinical practice: a meta-analysis of safety data. Stroke 2003; 34:2847-2850.

31 Marti-Fabregas J, Bravo Y, Cocho D, MartiVilalta JL, Diaz-Manera J, San Roman L, et al: Frequency and predictors of symptomatic intracerebral hemorrhage in patients with ischemic stroke treated with recombinant tissue plasminogen activator outside clinical trials. Cerebrovasc Dis 2007;23:85-90.

32 Scott PA, Frederiksen SM, Kalbfleisch JD, Xu Z, Meurer WJ, Caveney AF, et al: Safety of intravenous thrombolytic use in four emergency departments without acute stroke teams. Acad Emerg Med 2010;17:1062-1071.

33 Dzialowski I, Pexman JH, Barber PA, Demchuk AM, Buchan AM, Hill MD, et al: Asymptomatic hemorrhage after thrombolysis may not be benign: prognosis by hemorrhage type of the Canadian Alteplase for Stroke Effectiveness Study registry. Stroke 2007;38: 75-79.

34 Berger C, Fiorelli M, Steiner T, Schabitz WR, Bozzao L, Bluhmki E, et al: Hemorrhagic transformation of ischemic brain tissue: asymptomatic or symptomatic? Stroke 2001; 32:1330-1335.

35 Kohrmann M, Juttler E, Fiebach JB, Huttner HB, Siebert S, Schwark C, et al: MRI versus CT-based thrombolysis treatment within and beyond the $3 \mathrm{~h}$ time window after stroke onset: a cohort study. Lancet Neurol 2006;5: 661-667. 